Acta Phys. Hung. A / () -

HEAVY ION

PHYSICS

\title{
The role of noise and dissipation in the hadronization of the quark-gluon plasma
}

\author{
Eduardo S. Fraga ${ }^{1}$ \\ ${ }^{1}$ Instituto de Física, Universidade Federal do Rio de Janeiro \\ C.P. 68528, Rio de Janeiro, RJ 21941-972, Brazil \\ Received
}

\begin{abstract}
We discuss the role of noise and dissipation in the explosive spinodal decomposition scenario of hadron production during the chiral transition after a high-energy heavy ion collision. We use a Langevin description inspired by nonequilibrium field theory to perform real-time lattice simulations of the behavior of the chiral fields. Preliminary results for the interplay between additive and multiplicative noise terms, as well as for non-Markovian corrections, are also presented.
\end{abstract}

Keywords: Chiral transition; Heavy-ion collisions; Dissipation in field theory PACS: 25.75.-q, 11.30.Rd, 05.70.-a, 64.90.+b

\section{Introduction}

For high enough values of temperature, strongly interacting matter should be in a quark phase due to asymptotic freedom. In fact, finite-temperature lattice QCD simulations provide strong evidence of a deconfined quark-gluon plasma (QGP) phase at sufficiently high temperature [1]. Moreover, relativistic high-energy heavy ion collisions can probe strongly interacting matter under extreme conditions. They provide valuable information on the new state of matter that seems to have been created according to recent data from experiments at BNL-RHIC [ 2], even if its true nature is still uncertain.

As the QGP presumably created in a heavy-ion collision expands, it cools down and, at $T_{c} \sim 150 \mathrm{MeV}$ according to the Lattice [1], undergoes a phase transition (or a crossover) back to hadronic matter. Results from CERN-SPS and BNL-RHIC feature what has been called sudden hadronization [3] or explosive behavior [4] in the hadronization process of the expanding QGP and seem to favor a fast (explosive) spinodal decomposition scenario as the mechanism of phase conversion. This should 
be confronted to the process of bubble nucleation, whose time scales are larger due to the existence of a barrier to overcome [ [5]. Possible signatures of the explosive behavior in high-energy nuclear collisions were proposed in Ref. [6].

Effective field theory models for the chiral and the deconfinement transitions in QCD also indicate that most of the plasma is quenched into the spinodal region, due to the fast expansion of the system as compared to the low nucleation rate, then undergoes an explosive phase conversion [4, 7, 8, 9, 10, 11]. This leads to what we will refer to as the explosive spinodal decomposition scenario. Although an expansion term in the evolution equation for the order parameter plays a role somewhat similar to dissipation, most studies do not take into account genuine dissipative effects due to the interactions with the medium. However, dissipation effects have proved to be important, for instance, in the context of disoriented chiral condensate (DCC) formation in heavy ion collisions [12, 13, 14, 15, 16, 17] and could be an important ingredient in the explosive spinodal decomposition scenario.

Recently, we have considered the effects of dissipation on hadron production during the QCD transition after a high-energy heavy ion collision in the simplest fashion [18]. Using a phenomenological Langevin description for the time evolution of the order parameter, inspired by microscopic nonequilibrium field theory results [13, 14, 19, 20], we performed real-time lattice simulations for the behavior of the inhomogeneous chiral field. We concluded that the effects of dissipation could be dramatic even for very conservative assumptions. It was shown that even if the system quickly reaches this unstable region there is still no guarantee that it will explode.

Nevertheless, the description implemented in Ref. [18] captures only a small part of the much richer spectrum of possibilities for dissipation and noise effects in the dynamics of phase transitions. Part of this richness is captured by a linear response nonequilibrium quantum field theory analysis, which usually provides not only different contributions to noise and dissipation terms, but also complicated memory kernels instead of simple Markovian terms proportional to the time derivative of the field [13, 14, 19, 20].

In what follows, we present our findings from a traditional Langevin analysis of the evolution of the order parameter for the chiral transition, and discuss preliminary results including multiplicative noise [21] and memory effects [[22]. A robust feature that comes about from this study is that all the contributions tend to add up to delay the process of thermalization.

\section{Dissipation versus explosive decomposition}

We consider the real-time dynamics of chiral symmetry breakdown of a QGP created in a high-energy heavy ion collision [18]. We assume the system to be characterized by a coarse-grained free energy

$$
F(\phi, T)=\int d^{3} x\left[\frac{1}{2}(\nabla \phi)^{2}+V_{e f f}(\phi, T)\right],
$$


where $V_{\text {eff }}(\phi, T)$ is an effective potential of the Landau-Ginzburg form whose coefficients depend on the temperature, and $\phi(\vec{x}, t)$ is a real scalar field which plays the role of an order parameter that is not conserved, such as the chiral condensate. To model the mechanism of chiral symmetry breaking found in QCD, we adopt the linear $\sigma$-model coupled to quarks, whose standard Lagrangian can be found, for instance, in Ref. [ 10]. This approach is widely used in the literature and its specificities imply no major limitations to our main results. Quarks are integrated out yielding the effective potential $V_{\text {eff }}(\phi, T)$. The pion directions play no major role in the process of phase conversion we are considering, so we focus on the sigma direction represented by the field $\phi[$ [10]. For simplicity, we ignore effects due to the finite size of the plasma [23].

The framework for the dynamics is assumed to be given by the following Langevin equation:

$$
\frac{\partial^{2} \phi}{\partial t^{2}}-\nabla^{2} \phi+\eta \frac{\partial \phi}{\partial t}+\frac{d V_{\mathrm{eff}}(\phi)}{d \phi}=\xi(\vec{x}, t),
$$

where $\phi$ is a real scalar field and $\eta$, which can be seen as a response coefficient that defines time scales for the system and encodes the intensity of dissipation, is usually taken to be a function of temperature only, $\eta=\eta(T)$. The function $\xi(\vec{x}, t)$ represents a stochastic (noise) force, assumed Gaussian and white, so that $\langle\xi(\vec{x}, t)\rangle=0$ and $\left\langle\xi(\vec{x}, t) \xi\left(\overrightarrow{x^{\prime}}, t^{\prime}\right)\right\rangle=2 \eta T \delta\left(\vec{x}-\overrightarrow{x^{\prime}}\right) \delta\left(t-t^{\prime}\right)$, according to the fluctuation-dissipation theorem.

In our numerical simulations we solve Eq. (2) on a cubic space-like lattice with $64^{3}$ sites under periodic boundary conditions, with a lattice spacing of $a=0.91 \mathrm{fm}$. We use a semi-implicit finite-difference scheme for the time evolution and a Fast Fourier Transform for the spatial dependence [24]. Temperature is fixed to the spinodal value $T_{s p} \approx 108 \mathrm{MeV}$ [10]. We perform several runs starting from different random initial configurations around the inflexion point of $V_{\text {eff }}$ which happens at $\phi_{0} \approx 0.162 T$ and then average the results from the different initial configurations. For time steps of $\Delta t=0.001 / T$ the results become independent of the lattice spacing once it is smaller than $a \simeq 1 \mathrm{fm}$.

We show results of simulations for three different values of the dissipation coefficient, namely $\eta / T=0,2$ and 4 . It can be argued that the response coefficient has the form $\eta(T) \approx 2 T / b$, where $b$ is a number of order one to first approximation [ [25. The cases considered provide a conservative band around the value $\eta(T) \approx 2 T$ to illustrate the effect of dissipation.

In Figure 1we show the average value of $\phi$ in units of its vacuum value, $\phi_{v a c}$, as a function of time for the three different values of $\eta$ mentioned above. The results clearly show that even for a very conservative value of dissipation, $\eta=2 T$, the effect can be dramatic. For this value of $\eta$, dissipation retards the time evolution of $\phi$ towards its vacuum value in $\sim 100 \%$ compared to the case with $\eta=0$. The important point to be noted here is that for expansion times of the order of $5 \mathrm{fm} / \mathrm{c}$, which is of the order of the time scales for RHIC collisions, there might be not enough time for the onset of the spinodal explosion. 


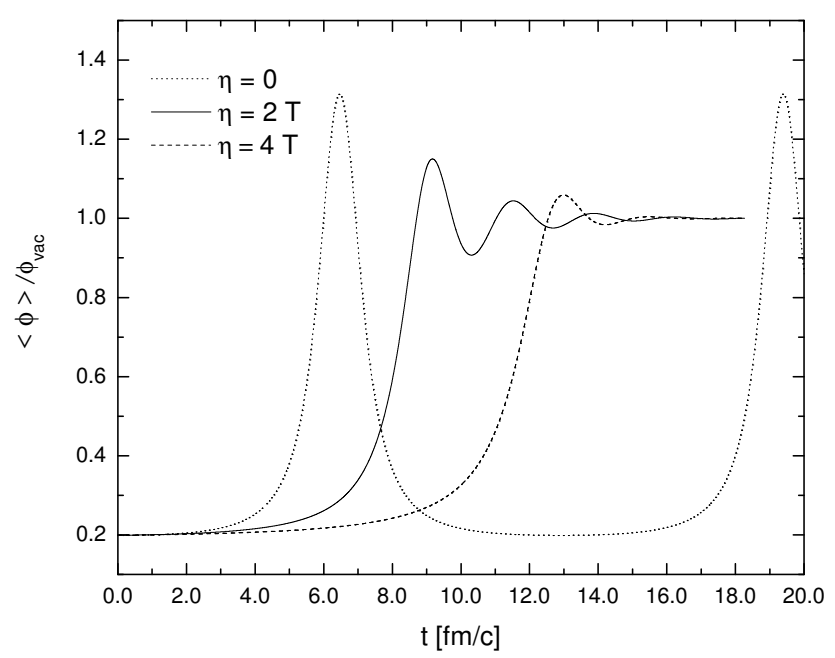

Fig. 1. Average value of the chiral field $\phi$ in units of its vacuum value $\phi_{v a c}$ as a function of time for $\eta / T=0,2,4$.

\section{Improved Langevin approach}

Although phenomenological equations of the form of Eq. (2) are widely used in the literature, formal derivations of effective equations of motion based on a more complete field-theoretic description of nonequilibrium dissipative dynamics [14, 19, 20] show that much more complicated equations emerge. In general they are non-local equations of motion, with colored, non-Markovian dissipative and noise kernels, exhibiting both noise and dissipation terms that depend on the field amplitude. It is then important to analyze the possible outcome of dynamics emerging from the more realistic equations as compared to the case of the simpler phenomenological equation (2). In fact, on very general physical grounds, one expects that dissipation effects should depend on the local density $\sim \phi^{2} \dot{\phi}$ and, accordingly, the noise term should contain a multiplicative piece $\sim \phi$. These results emerge as approximations to the effective equations of motion derived e.g. in Refs. [14, 19, 20].

Motivated by the results referred above, we consider an improved Langevin framework which includes the effects of multiplicative noise and density-dependent dissipation terms in the description of the time evolution of the order parameter. To assess the effect of the new contributions on the relevant time scales for phase ordering, one can adopt a scalar $\lambda \phi^{4}$ theory in the broken phase, whose standard Lagrangian density has the form:

$$
\mathcal{L}=\frac{1}{2}\left(\partial_{\mu} \phi\right)\left(\partial^{\mu} \phi\right)+\frac{1}{2} m^{2} \phi^{2}-\lambda \phi^{4},
$$


where $\phi$ is a scalar field playing the role of the order parameter and $\lambda>0$. We perform $(3+1)$-dimensional real-time lattice simulations to study the behavior of the inhomogeneous scalar field, taking into account lattice counterterms that guarantee lattice-size independence [15, 26, 27].

To define an initial value problem for the evolution of the order parameter, one can assume that the system was quenched from a high-temperature environment in which the sign of the mass term was the opposite, corresponding to a symmetric phase, down to a temperature below some critical temperature of symmetry restoration $\left(T<T_{c}\right)$, in which the effective potential has the form shown in the Lagrangian density 3

In our analysis, the time evolution of the field $\phi(\vec{x}, t)$ at each point in space and its approach to equilibrium will be dictated by an improved Langevin equation of the form

$$
\left(\frac{\partial^{2}}{\partial t^{2}}-\nabla^{2}\right) \phi(\vec{x}, t)+\left[\eta_{1}+\eta_{2} \phi^{2}(\vec{x}, t)\right] \frac{\partial \phi(\vec{x}, t)}{\partial t}+V^{\prime}(\phi)=\xi_{1}(\vec{x}, t)+\phi(\vec{x}, t) \xi_{2}(\vec{x}, t),
$$

which models the local approximation for the effective equation of motion derived e.g. in Ref. [19. In Eq. (4), $\eta_{1}$ and $\eta_{2}$ will be taken to be functions of temperature only, $\eta_{i}=\eta_{i}(T)$. The functions $\xi_{1}(\vec{x}, t)$ and $\xi_{2}(\vec{x}, t)$ represent stochastic (noise) forces, assumed Gaussian and white.

Eq. (44) could, in principle, be obtained from a microscopic field-theoretic description of the real-time nonequilibrium dynamics of the chiral field at finite temperature [14, 19]. The noise and dissipation terms, which originate from quantum fluctuations, are engendered by either self-interactions of the chiral field or coupling to one or more different fields that play the role of a heat bath, provided one incorporates higher-order terms in the computation of the effective equation of motion for $\phi(\vec{x}, t)$. In fact, it is well-known that one has to go up to two-loop corrections in order to pick up imaginary parts in the self-energy associated with viscosity and dissipation [14, 16, 19, 20]. Self-interactions of the $\phi$ field, as well as possible interactions with other fields in the medium, fully justify the inclusion of dissipation and noise terms such as done in the framework adopted here.

In our numerical simulations we solve Eq. (4) on a cubic space-like lattice. We use the leap frog method (see e.g. Refs. [26, 27]) for the time evolution and treat the Laplacian using a Fast Fourier Transform for the spatial dependence [ 24]. To minimize lattice artifacts, we use counterterms calculated in lattice perturbation in the classical theory (tadpole plus setting-sun diagrams) [ 28]:

$$
V_{C T}=\frac{1}{2}\left\{-3 \times 0.252731 \frac{T}{a}+\frac{6 T^{2}}{(4 \pi)^{2}}\left[\log \left(\frac{6}{\mu a}\right)+0.009\right]\right\} \phi^{2},
$$

where $T$ is the temperature, $a$ is the lattice spacing and $\mu$ is an arbitrary renormalization scale. It should be noted that the addition of this counterterm is supposed to guarantee independence of the results on $a$ at equilibrium only. We checked the lattice independence of the results [21], and adopt units such that $m=1$ in 


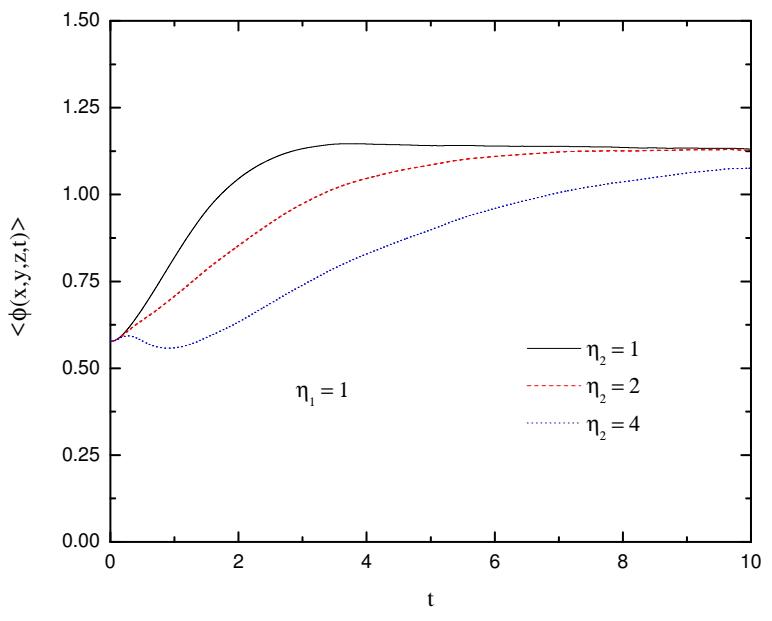

Fig. 2. Average value of the field $\phi$ as a function of time under the effect of additive and multiplicative noises $\left(\eta_{1}=1\right.$ and $\left.\eta_{2}=1,2,4\right)$

the Lagrangian of Eq. (3), i.e. all dimensional quantities are expressed in units of $m$. In our simulations, the lattice extent is fixed to $L=16$, and the time step is $\Delta t=10^{-3} ; T=1, \lambda=1$, and $\mu=1$. The initial configuration is taken to be a random distribution of the form $\phi(x, t)=\phi_{s p}+0.001 * \operatorname{rand}($ seed $)$, where $\phi_{s p}=1 / \sqrt{3}$ is the spinodal point of the bare double-well potential, and $\mathrm{rand}(\mathrm{seed})$ is a uniform random number distribution in the interval $[-1,1]$. At the end, results are averaged over several runs for different realizations of the noises.

In Figure 2 we present preliminary results for the volume average of $\phi$ as a function of time for different values of the multiplicative dissipation parameter $\eta_{2}$. For simplicity, $\eta_{1}$ and $\eta_{2}$ are taken to be constants. More realistic parametrizations will appear soon [21]. As $\eta_{2}$ increases, the effect is of delaying the equilibration of the order parameter, as expected.

\section{Non-Markovian corrections}

As discussed before, the structure of memory integrals and colored noise that appear in a realistic field-theoretic description of the dynamics of phase transitions is often rather complicated [ 29, 30]. To develop and test new approaches, one can consider the much simpler case of dissipation in quantum mechanics, where all the approximations and important scales are under control, and where one also finds inumerous applications [31]. 
Starting from the nonequilibrium evolution of a particle coupled linearly to a set of harmonic oscillators in the Caldeira-Leggett fashion [ 31], we study the effects of the non-local dissipation kernel, as well as of colored noise, that appear in the complete Langevin equation for the particle coordinate in space [ 22]. There, the memory kernel has its origin in the Feynman influence functional of the heat bath. To approach the kernel in a simpler, analytic way, we develop a systematic expansion in time derivatives whose convergence is regulated by increasing powers of the frequency cutoff in the distribution of oscillators, $\Omega$. Using this method, one can incorporate consistently corrections coming from the memory integral. The equation of motion thus obtained has the following form [22]:

$$
M \ddot{Q}+V^{\prime}(Q)+\frac{2 \eta}{\pi} \sum_{n=0}^{\infty} \frac{I_{n}(\Omega t)}{n !} \frac{Q^{(n+1)}(t)}{\Omega^{n}}=\xi(t),
$$

which reduces to the traditional Langevin equation with white noise in the limit $\Omega t \rightarrow \infty$, since $I_{n}(\infty) \rightarrow \delta_{n 0} \pi / 2$ and the noise correlator tends to $2 \eta T \delta\left(t-t^{\prime}\right)$, consistently with the fluctuation-dissipation theorem. $I_{n}(\Lambda)$ are integral coefficients which can be expressed in terms of incomplete gamma functions. Inspection of ([6) shows that terms containing higher-order time derivatives of $Q$ are strongly suppressed by increasing powers of $1 / \Omega$.

\section{Final remarks}

The construction of a microscopic field-theoretic framework to study the role of noise and dissipation in a realistic, yet efficient, way is still not accomplished. We believe this task will require the development of systematic (controlled) analytic approximations to simplify memory kernels, as well as effective numerical methods to deal with arbitrary colored noise. In the case of the chiral transition in heavy ion collisions, effects brought about by the expansion of the plasma [4] and by its finite size [23] will also bring corrections to this picture. Some of these issues will be addressed in future publications [[21, 22].

\section{Acknowledgments}

My special thanks to T. Kodama, G. Krein, L. F. Palhares and R. O. Ramos for stimulating discussions and collaboration. The author also thanks the organizers of the QGP Thermalization Workshop for providing such a nice environment for the debate of ideas. The work of E.S.F. is partially supported by CAPES, CNPq, FAPERJ, and FUJB/UFRJ.

\section{References}

1. E. Laermann and O. Philipsen, Ann. Rev. Nucl. Part. Sci. 53 (2003) 163. 
2. Proceedings of Quark Matter 2004, J. Phys. G 30 (2004) S633.

3. J. Rafelski and J. Letessier, Phys. Rev. Lett. 85 (2000) 4695.

4. A. Dumitru and R. D. Pisarski, Nucl. Phys. A 698 (2002) 444; O. Scavenius, A. Dumitru and A. D. Jackson, Phys. Rev. Lett. 87 (2001) 182302.

5. J. D. Gunton, M. San Miguel and P. S. Sahni, in: Phase Transitions and Critical Phenomena, Eds. C. Domb and J. L. Lebowitz, Academic Press, London, 1983, v. 8.

6. J. Randrup, Phys. Rev. Lett. 92 (2001) 122301; arXiv:nucl-th/0406031

V. Koch, A. Majumder and J. Randrup, arXiv:nucl-th/0509030

7. R. D. Pisarski, Phys. Rev. D 62 (2000) 111501; A. Dumitru and

R. D. Pisarski, Phys. Lett. B 504 (2001) 282.

8. L. P. Csernai and I. N. Mishustin, Phys. Rev. Lett. 74, 5005 (1995).

9. O. Scavenius and A. Dumitru, Phys. Rev. Lett. 83, 4697 (1999).

10. O. Scavenius et al., Phys. Rev. D 63, 116003 (2001).

11. K. Paech, H. Stocker and A. Dumitru, Phys. Rev. C 68, 044907 (2003).

12. T. S. Biro and C. Greiner, Phys. Rev. Lett. 79 (1997) 3138.

13. C. Greiner and B. Muller, Phys. Rev. D 55, 1026 (1997).

14. D. H. Rischke, Phys. Rev. C 58 (1998) 2331.

15. L. M. A. Bettencourt, K. Rajagopal and J. V. Steele, Nucl. Phys. A 693 (2001) 825.

16. A. Mocsy, Phys. Rev. D 66 (2002) 056010.

17. G. Holzwarth and J. Klomfass, Phys. Rev. D 66 (2002) 045032.

18. E. S. Fraga and G. Krein, Phys. Lett. B 614, 181 (2005).

19. M. Gleiser and R. O. Ramos, Phys. Rev. D 50 (1994) 2441.

20. A. Berera, M. Gleiser and R. O. Ramos, Phys. Rev. D 58, 123508 (1998).

21. E. S. Fraga, G. Krein and R. O. Ramos, to appear.

22. E. S. Fraga, T. Kodama, G. Krein and L. F. Palhares, to appear.

23. E. S. Fraga and R. Venugopalan, Physica A 345 (2004) 121.

24. M.I.M. Copetti and C.M. Elliot, Mat. Sci. Tecn. 6 (1990) 273.

25. K. Kajantie, Phys. Lett. B 285 (1992) 331.

26. J. Borrill and M. Gleiser, Nucl. Phys. B 483, 416 (1997).

27. C. J. Gagne and M. Gleiser, Phys. Rev. E 61, 3483 (2000).

28. K. Farakos et al., Nucl. Phys. B 425, 67 (1994).

29. A. Arrizabalaga, J. Smit and A. Tranberg, Phys. Rev. D 72, 025014 (2005).

30. J. Berges and I. O. Stamatescu, arXiv:hep-lat/0508030

31. U. Weiss, Quantum Dissipative Systems, $2^{\text {nd }}$ edition (World Scientific, 1999). 